\title{
EFFICIENCY OF THE CONSTANT INTERACTION FORCE VIBROISOLATION (WOSSO)
}

\author{
MARIAN Witalis DobRY \\ Poznan University of Technology, Institute of Applied Mechanics, Poznań, Poland \\ e-mail: marian.dobry@put.poznan.pl
}

\begin{abstract}
In this paper, the force efficiency of the WoSSO vibroisolation in reducing forces transferred into the base by machines and the equipment working at low operating frequencies has been discussed. To assess that efficiency, a strongly non-linear mathematical model using Lagrange equations of the second kind has been developed. That model takes into account the specific design of the vibroisolator. The mathematical model has been solved using s digital simulation method, by developing a special computer program in the MATLAB/simulink environment. The efficiency of the force vibroisolation has been determined for a specific application of the WoSSO vibroisolator. The calculated force vibroisolation efficiency exceeds 32 .
\end{abstract}

Keywords: low-frequency passive vibroisolation, efficiency of force vibroisolation

\section{Introduction}

All vibration reduction methods still aim at ensuring effective passive vibroisolation. Practical use of common spring, rubber and pneumatic vibroisolators is very difficult in the case of vibroisolation from low-frequency excitations generated by vibration sources. Each passive vibroisolation is characterised by a specific vibroisolation efficiency limit value. That value depends on the resonance of the vibroisolating system and a (limited) strength of the materials used for production of the vibroisolators. A vibroisolating system is characterised by a very low frequency of proper vibrations. That requires large deflections of vibroisolators - which, in turn, ensures large dynamic flexibility of the vibroisolation (Goliński, 1979; Harris and Crede, 1976).

That difficulty has been eliminated in the Constant Interaction Force Vibroisolation system (WoSSO) developed in the Division of Vibroacoustics and Biodynamics of Systems at the Institute of Mechanics of Poznan University of Technology (Dobry, 1983, 1998; Dobry and Cempel, 1983).

The substitute spring constant coefficient of that vibroisolation is zero and independent of the deflection of the vibroisolating system. The stability of characteristics makes that kind of vibroisolation better than the pneumatic one, the characteristic of which is stiff (exponential) at large deflections.

In this paper, the application of the WoSSO vibroisolation to force vibroisolated machines and equipment has been discussed. An important parameter for the assessment of the efficiency of that specific application of the WoSSO vibroisolation is the force efficiency of the vibroisolation at low-frequency vibrations.

The efficiency of the WoSSO in application to free masses (for example machines) has not yet been determined.

Furthermore, the analyzed vibroisolation system has been supplemented with an additional spring $k_{1}$ for faster stabilization of motion of the mass $M$ - see Fig. 1. This spring has been also used to reduce the deflection from the static equilibrium position defined for the beginning of the motion. 


\section{Design of the WoSSO vibroisolator}

The WoSSO vibroisolation is used for vibroisolating from forces generated by a machine in operation. It protects the base against strong and variable forces transferred into the base, which takes place if the vibroisolation is not used. The design of such type of WoSSO vibroisolation is presented in Fig. 1 (Dobry, 1983, 1998; Dobry and Cempel, 1983).

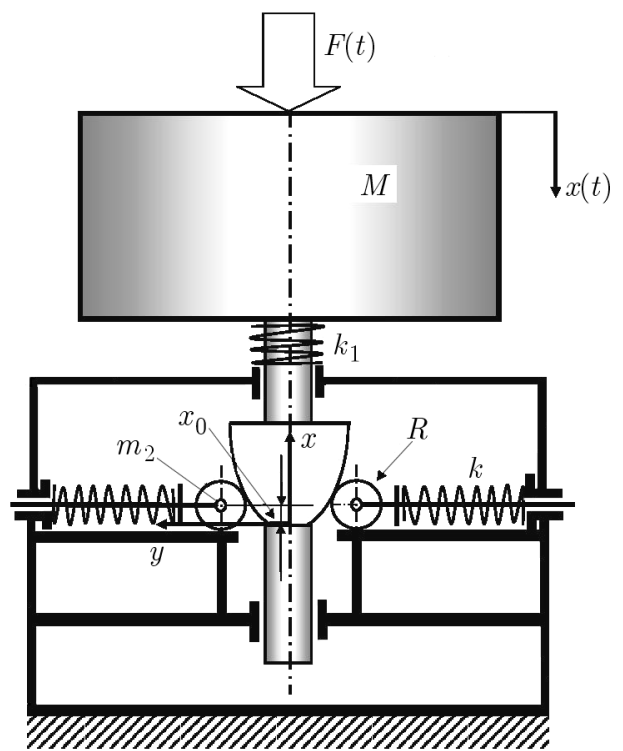

Fig. 1. Design of the Constant Interaction Force Vibroisolator (WoSSO) for force vibroisolation supplemented with an additional spring $k_{1}$

The properties of the WoSSO vibroisolation are discussed on the basis of the WoSSO vibroisolator which is used to vibroisolation of the base from forces exciting the mass $M$ to move. It is composed of a cam mounted on a working pin guided in the slide bearings mounted in the vibroisolator body. That cam has been pressed in between two pressure rollers with radius $R$. Those rollers are guided by two guiding rollers mounted at both sides of the pressure rollers (these guiding rollers may also have the same radius $R$ ) and by a special guiding shackle. The rollers are pressed against the cam by two vibroisolator springs (with the spring constant $k$ ) preliminary compressed with the force $S_{0}$. The shape of the cam curve is the same as the curve of motion of the axis of the pressure and guiding rollers (Dobry, 1983, 1998, Dobry and Cempel, 1983)

$$
y=\frac{-S_{0}+\sqrt{S_{0}^{2}+P k x_{0}+P k x}}{k}
$$

where $S_{0}$ is the vibroisolator spring preliminary compression force, $P$ - constant force of vibroisolator reaction - it is equal to the weight of mass $M$, working pin and cam, $k$-vibroisolator spring constant, $x$ - generalized co-ordinate that describes vertical motion of the reduced mass $M, x_{0}$ - initial position of mass $M$.

The co-ordinate system used to describe the cam is shown in Fig. 1. The position (deflection) of the vibroisolator at the moment $t=0$ is defined by the co-ordinate $x_{0}$ of the initial position.

\section{Mathematical model of the analysed mechanical system comprising the WoSSO vibroisolator}

The mathematical model of the analysed system comprising the WoSSO vibroisolator has been prepared using Lagrange's equation of the second kind (Cannon, 1973) 


$$
\frac{d}{d t}\left(\frac{\partial E}{\partial \dot{q}_{j}}\right)-\frac{\partial E}{\partial q_{j}}=\frac{\delta L_{j}}{\delta q_{j}}-\frac{\partial V}{\partial q_{j}}-\frac{\partial \Phi}{\partial \dot{q}_{j}} \quad j=1,2, \ldots, s
$$

where $s$ is the number of degrees of freedom, $E$ - kinetic energy of the mechanical system, $q_{j}$ - generalized co-ordinates, $\dot{q}_{j}$ - generalized velocities, $\delta L_{j}$ - small increment of the virtual work at the $j$-th virtual displacement along the direction not limited by constraints, $\delta q_{j}$ - small increment of the $j$-th generalized co-ordinate along the direction not limited by constraints, $V-$ potential energy of the mechanical system, $\Phi$ - power of energy dissipation in the mechanical system.

When calculating the kinetic energy, the virtual work of the external active forces, the potential energy and the power of energy losses and by making the mathematical operations defined by Lagrange's equations (3.1), a single equation of forces has been obtained as the analysed mechanical system has one degree of freedom connected with motion ot the mass $M$. The final equation of force is in form

$$
\begin{aligned}
(M & \left.+\frac{0.5 P^{2} m_{2}}{S_{0}^{2}+P k x_{0}+P k x}\right) \ddot{x}-\frac{0.75 P^{3} k m_{2}}{\left(S_{0}^{2}+P k x_{o}+P k x\right)^{2}} \dot{x}^{2} \\
& +\frac{f_{2}}{R}\left[\frac{f_{1}}{r} P \operatorname{sgn}(\dot{x})+2 \sqrt{S_{0}^{2}+P k x_{0}+P k x}\right] \operatorname{sgn}(\dot{x})+k_{1} x=F_{0} \sin \left(2 \pi f_{w} t\right)
\end{aligned}
$$

where $x(t)=q_{1}(t)$ is the coordinate of the position of the mass $M, k_{1}$ - spring stiffness for faster stabilization of motion of the mass $M$ of a low value, $m_{2}$ - total mass as a sum of masses of pressure rollers, guiding rollers, axis and the guide of the bearings shackle.

The above differential equation of motion has been elaborated taking into account the following simplifying assumptions (taking into account the individual design and operation of the vibroisolator):

1) weight of the mass $M$ is equilibrated by the constant vibroisolator response force $P$ in the whole range of deflections,

2) kinetic energy does not comprise the mass of the vibroisolator springs,

3) kinetic energy does not comprise rotation of the rollers,

4) mass of the axis rollers and the roller guiding shackle in the related rectilinear motion along the $y$ direction depending on the cam shape has been taken into account,

5) variable rolling friction of the rollers along the cam and the guides has been taken into account,

6) the following structural frictions have not been taken into account: the structural friction inside the rollers shackle guides, in the rollers bearings and in the slide bearings of the working pin,

7) it has been assumed that the system is excited by a harmonic force with the amplitude $F_{0}$ and frequency $f_{W}[\mathrm{~Hz}]$.

The above assumptions have been made considering a negligible impact of disregarded phenomena and physical quantities on the motion of the analysed system. Strongly non-linear effects have been taken into account in the above differential equation of forces (Minorski, 1967). Those effects refer to the structural friction of the vibroisolator for the rolling pressure and guiding rollers with such friction being dependent on the square of the preliminary compression force of the vibroisolator springs $k$ and on the sign of velocity of the mass $M$. The variability of the inertial force during motion of the mass $M$ is also shown in the equation (that variability is dependent on the deflection of the vibroisolator $x(t)$ ). The system also generates strongly non-linear forces which depend on the square of the velocity of the mass $M$, on the second and third square of the permanent vibroisolator reaction force $P$ and on the deflection of the vibroisolator $x(t)$ along its operating direction. 
Dividing equation (3.2) by the dynamic parameter of mass $B$

$$
B=M+\frac{0.5 P^{2} m_{2}}{S_{0}^{2}+P k x_{0}+P k x}
$$

a differential equation (3.4) of motion of the mass $M$ has been obtained. The equation has been solved assuming the following initial conditions: $x(0)=0.015 \mathrm{~m}$ and velocity $v(0)=0 \mathrm{~m} / \mathrm{s}$

$$
\begin{aligned}
\ddot{x}- & \frac{0.75 P^{3} k m_{2}}{B\left(S_{0}^{2}+P k x_{0}+P k x\right)^{2}} \dot{x}^{2}+\frac{f_{2}}{R B}\left[\frac{f_{1}}{r} P \operatorname{sgn}(\dot{x})+2 \sqrt{S_{0}^{2}+P k x_{0}+P k x}\right] \operatorname{sgn}(\dot{x}) \\
& +\frac{k_{1}}{B} x=\frac{F_{0}}{B} \sin \left(2 \pi f_{w} t\right)
\end{aligned}
$$

Such complex form of the strongly non-linear equation does not have a closed-form pure equation solution (Cannon, 1973; Minorski, 1967). To determine motion of the mass $M$, a digital simulation of the solution to the differential equation of motion has been applied. For that purpose, a special software called SDWoSSO has been developed using the MATLAB/simulink software.

\section{Solution of the differential equation of motion for the mechanical system with the force vibroisolation WoSSO}

The structure of the computer program for simulation of the dynamics of the vibroisolator WoSSO (SDWoSSO) has been shwn in Figs. 2 and 3. Figure 2 shows the computer program for calculation of the efficiency of the force vibroisolation (EWS) of WoSSO, i.e. the ratio of the RMS values of the following forces: the force that excites vibrations of the mass $M$ (source of vibration $)-F_{w}(\mathrm{RMS})$ to the dynamic reactive force of the base $R_{p}(\mathrm{RMS})$ - the protected area. This efficiency has been expressed by

$$
\text { EWS_WoSSO }=\frac{F_{w}(\mathrm{RMS})}{R_{p}(\mathrm{RMS})}
$$

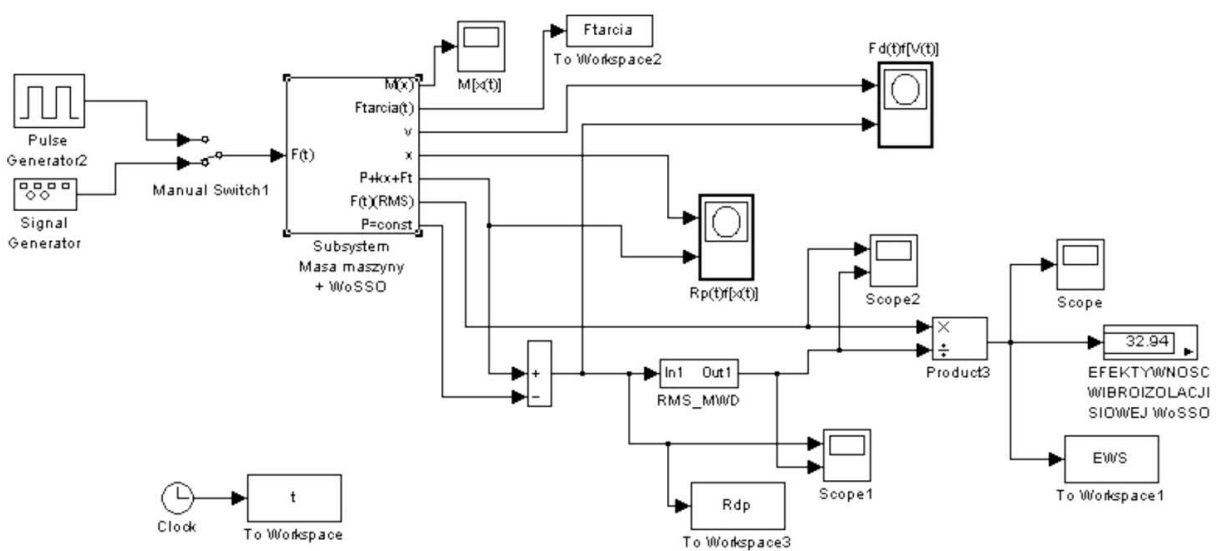

Fig. 2. Structure of the computer program for simulation of the dynamics of the force vibroisolation that uses the WoSSO vibroisolator - calculation of the efficiency

The computer program for digital simulation of the WoSSO vibroisolator dynamics enables tracking the efficiency during the system start-up, i.e. from a standstill until the steady-state motion. 


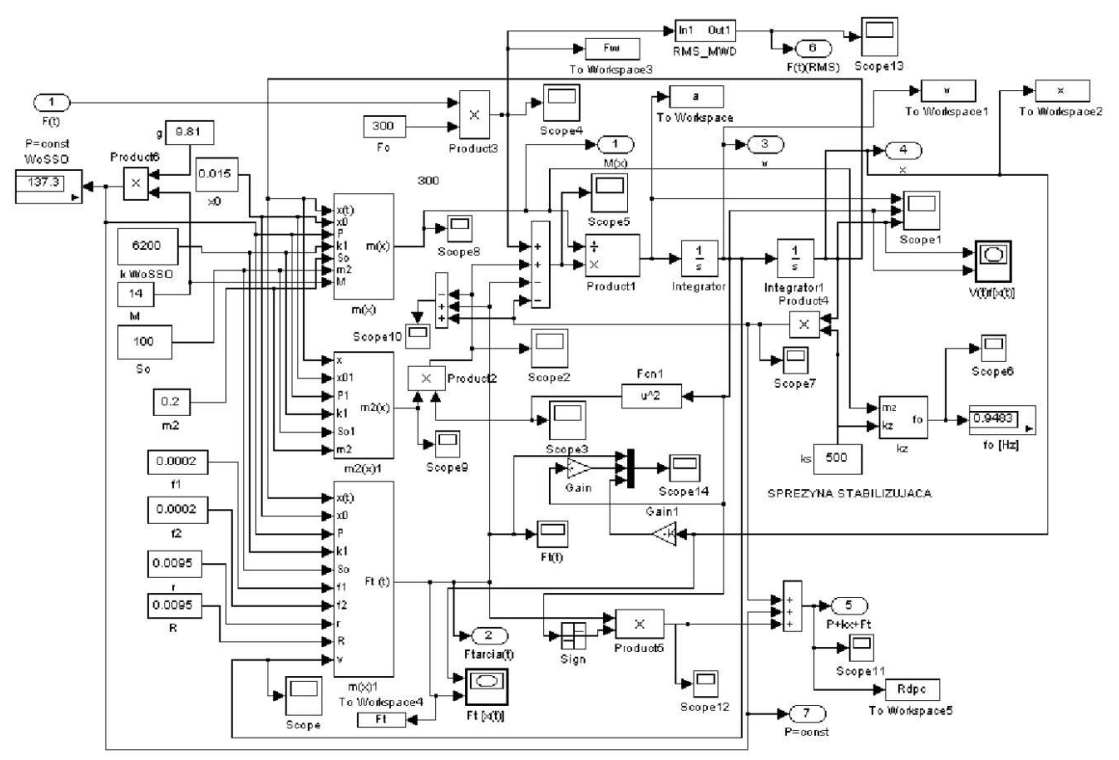

Fig. 3. Structure of the subprogram for simulation of the dynamics of the force vibroisolation that uses the WoSSO vibroisolator - simulation using the mathematical model

Figure 3 shows the structure of the subprogram for simulation of the dynamics of the force isolation that uses the WoSSO vibroisolator. That program solves, through simulation, the strongly non-linear mathematical model of the force vibroisolation that uses the WoSSO vibroisolator.

The following data (from practical use of the WoSSO vibroisolator) have been used in sample calculations: $P=137.3 \mathrm{~N}, k=6500 \mathrm{~N} / \mathrm{m}, M=14 \mathrm{~kg}, S_{0}=100 \mathrm{~N}, x_{0}=0.015, m_{2}=0.2 \mathrm{~kg}$, $f_{1}=f_{2}=0.0002, R=0.0095 \mathrm{~m}, k_{1}=500 \mathrm{~N} / \mathrm{m}, F_{0}=300 \mathrm{~N}, f_{w}=16 \mathrm{~Hz}$, simulation time $t=120 \mathrm{~s}$.

In the paper, the calculation results have been presented for the following case: the sinusoidal pattern of the exciting force characterised by the amplitude $F_{0}=300 \mathrm{~N}$ and the frequency $16 \mathrm{~Hz}$ applied to the concentrated mass $M=14 \mathrm{~kg}$ placed on the WoSSO vibroisolator.

In Figs. 4a,b,c the acceleration, velocity and displacement of the reduced mass $M$ for a sinusoidal exciting force with amplitude $300 \mathrm{~N}$ are presented. The acceleration amplitude reaches $21.8 \mathrm{~m} / \mathrm{s}^{2}$, the velocity amplitude $0.21 \mathrm{~m} / \mathrm{s}$, and the mass $M$ displacement is $0.0021 \mathrm{~m}$.

Figure 5 shows the time history of the force of structural friction with amplitude $6.59 \mathrm{~N}$.

The variability of the friction force is caused by the resistance of the pressure rollers rolling along the guides and the resistance of the pressure rollers rolling along the WoSSO cam, which generate variable pressure forces during their motion. Figure 6 shows the structural friction force during the whole start-up procedure as a function of the displacement $x(t)$ of the mechanical system.

It shows the impact of variable pressure forces generated by the pressure rollers rolling along the cam. This variability results from changes in the cam shape for all cam positions $[(-) 0.025$ to approx. $0.0185 \mathrm{~m}$ ] taken during the transitional process, i.e. from time $t=0$ to $120 \mathrm{~s}$. The dense concentration of lines (shown in the figure) is the area with the variable friction force for steady-state motion of the analysed mechanical system.

\section{Force efficiency of the WoSSO vibroisolator}

An important parameter for assessing the vibroisolation is the vibroisolation efficiency. In the case in question, the WoSSO vibroisolator uses the force vibroisolation defined by formula (4.1). 

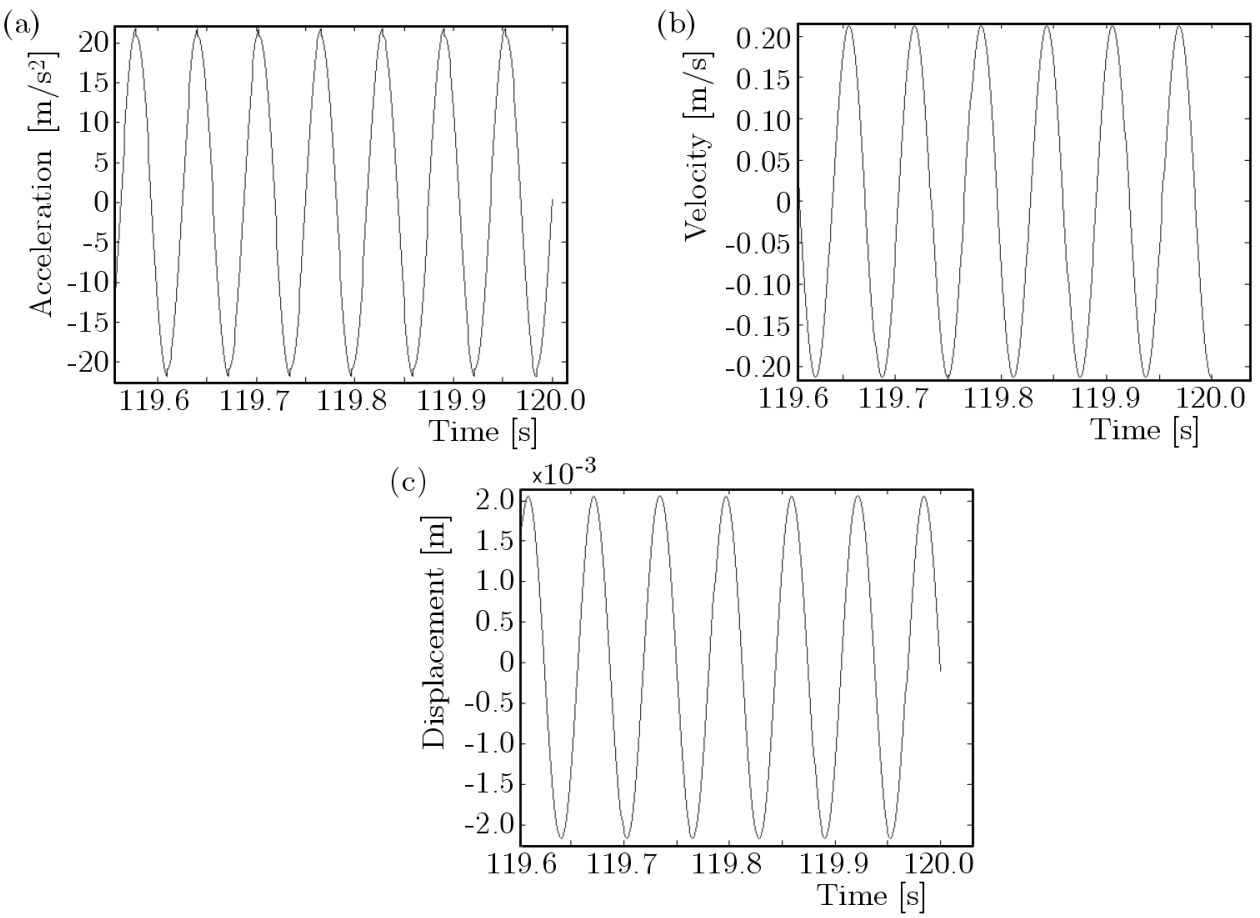

Fig. 4. Acceleration (a), velocity (b) and displacement (c) of the mass $M$ in steady-state motion of the mechanical system

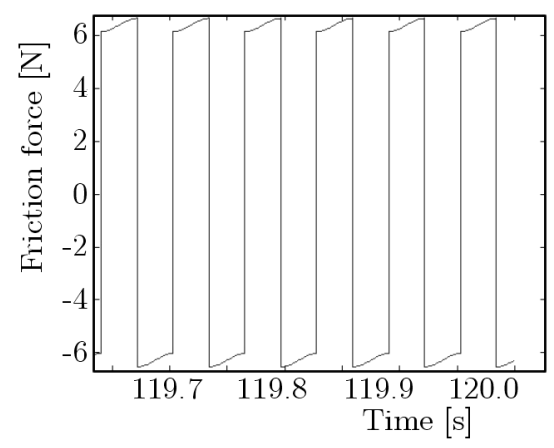

Fig. 5. Time history of the structural friction force in the WoSSO in steady-state motion of the mechanical system

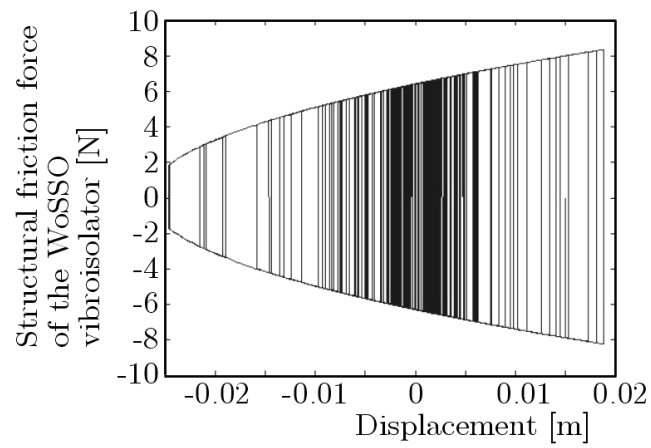

Fig. 6. Structural friction force pattern for the WoSSO vibroisolator as a function of the reduction point displacement during the start-up of the system comprising the WoSSO vibroisolation 
The force efficiency of the above vibroisolator has been calculated for the whole duration of the start-up phase of the mechanical system, from a standstill to the steady-state motion. To determine that efficiency, has been also calculated the dynamic base response. The latter was determined by subtracting, from the total base response, the constant WoSSO vibroisolator load capacity which equals the weight of mass $M$. The dynamic response of the base is shown in Fig. 7. As shown, the force of the response has a constant component of approx. $6.81 \mathrm{~N}$, and its minimum and the maximum value is 7.685 and $4.95 \mathrm{~N}$, respectively. The root-mean-square value (RMS) of the dynamic base response needed for calculation of the force efficiency of the WoSSO vibroisolator is $6.43 \mathrm{~N}$.

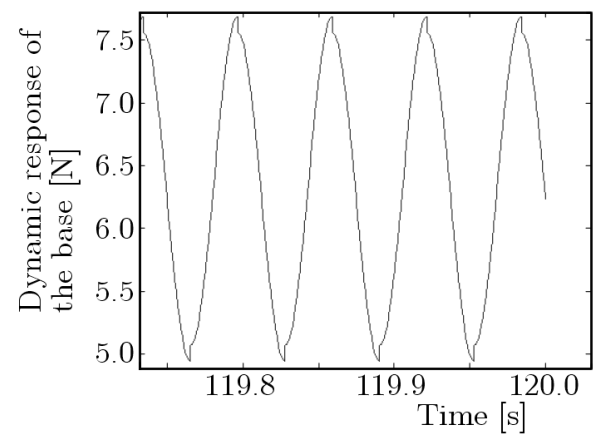

Fig. 7. Dynamic response of the base vibroisolated by WoSSO (steady-state motion)

The changes of the efficiency value of the force vibroisolation of the WoSSO vibroisolator during the start-up of the mechanical system are shown in Fig. 8. The final value of that efficiency for the steady-state motion after $120 \mathrm{~s}$ of the system dynamics simulation is 32.94 .

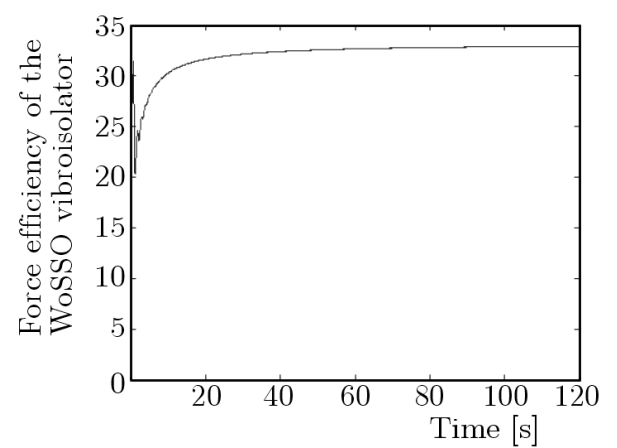

Fig. 8. Force vibroisolation efficiency of the WoSSO vibroisolator at the start-up of the mechanical system

It means that the force exciting motion of the mass $M$ is reduced by the vibroisolator by more than 32 times. The efficiency of the force vibroisolation is very high, given the fact that it is a passive vibroisolation.

The specific characteristic of this vibroisolator is that the vibroisolation effectiveness does not drop below one at the beginning of motion, which means that the force transferred into the base is amplified. On the contrary, the maximum value of the force efficiency of 31.5 is achieved within $0.71 \mathrm{~s}$ from the start of motion. It is a quite significant difference when compared to standard vibroisolators in which no vibroisolation effect is visible during the start-up phase (force is amplified), which is connected with passage of the system through the resonance.

The efficiency drop down to approx. 20.3 (as shown in Fig. 8) is caused by the stabilising spring $k_{1}$. Because of the impact of that spring, the natural frequency of the analysed mechanical system is equal to $0.94 \mathrm{~Hz}$ - the passage through the resonance results in a reduced efficiency of the vibroisolation. 


\section{Stability of motion of the WoSSO vibroisolator used for force vibroisolation}

Practical usefulness of strongly non-linear vibroisolators depends on the stability of motion of the mechanical system. For this purpose, a digital simulation of the dynamics of the system has been carried out to show the motion of the mass $M$ placed on the WoSSO vibroisolator on the phase plane (Fig. 9).

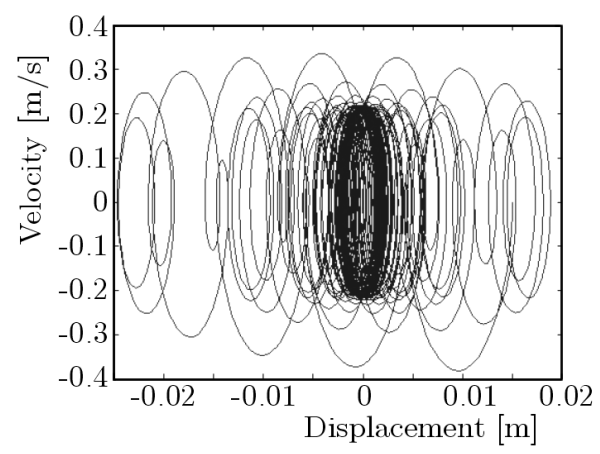

Fig. 9. Phase portrait of the transitional process for force vibroisolation that uses the WoSSO vibroisolator - stability of the motion

The whole transitional process of the mass $M$ motion from $t=0$ to $t=120 \mathrm{~s}$ has been shown. The phase portrait is a confirmation of the good stability of the mechanical system - the motion of mass $M$ tends to achieve a stable orbit in the steady-state motion. That result shows that using the WoSSO vibroisolator for force vibroisolation of various technical objects will not cause any implementation problems.

\section{Conclusions}

- The Constant Interaction Force Vibroisolator (WoSSO) supplemented with an additional spring $k_{1}$ is passive and strongly non-linear.

- The efficiency of the force vibroisolation is more than 32.9 for sample parameters used for calculations: mass $M=14 \mathrm{~kg}$, motion exciting force: sinusoidal pattern with amplitude $300 \mathrm{~N}$ and frequency $16 \mathrm{~Hz}$.

- Motion of the mass placed on the WoSSO vibroisolator supplemented with the additional spring $k_{1}$ is stable, therefore the WoSSO vibroisolator can be efficiently used to force vibroisolation of machines and the equipment.

The properties described above makes the WoSSO vibroisolation suitable for a wide range of applications. The tests on the vibroisolation efficiency in various applications will be continued.

\section{References}

1. Cannon R.H. JR., 1973, Dynamics of Physical Systems (in Polish), WNT, Warszawa

2. Dobry M.W., 1983, Dynamics and Stability of the Constant Interaction Force Vibroisolator for Hand-Held Percussive Tools (in Polish), Ph.D. Thesis, Poznan University of Technology, Department of Mechanical Engineering and Management, Poznań, Poland

3. Dobry M.W., 1998, Optimisation of the Energy Flow within the Human-Tool-Base System (in Polish), Wyd. Politechniki Poznańskiej, Poznań, Seria: Rozprawy Nr 330, ISSN 0551-6528 
4. Dobry M.W., Cempel C., 1983, Vibroisolator (in Polish), Patent RP nr 120 458, Urząd Patentowy RP. Opis patentowy opublikowano (published 25.07.1983)

5. Goliński J.A., 1979, Vibroisolation of Machines and Equipment (in Polish), WNT, Warszawa

6. Harris C.M., Crede C.E., 1976, Shock and Vibration Handbook, chapt. 30-33, McGraw-Hill, New York

7. Minorski N., 1967,Non-linear Vibrations (in Polish), PWN, Warszawa

Manuscript received November 13, 2013; accepted for print June 10, 2014 